\title{
Dual-Mode Circular Waveguide Filters Without Tuning Screws
}

\author{
M. Guglielmi, Member, IEEE, R. C. Molina, and A. Alvarez Melcon
}

\begin{abstract}
The manufacture of dual-mode circular waveguide filter is a complex and labor intensive task that could be greatly simplified with the use of accurate and fast CAD tools to predict the electrical behavior of the structures being developed. This is especially true for the "tuning screws assembly" that is an integral part of this type of filters. A method is proposed for the rigorous and efficient analysis of the tuning screws assembly for dualmode filters in circular waveguide. The method is based on the introduction of a new waveguide called ridged circular waveguide and in its use as a key component for the implementation of dual-mode filters. The modal spectrum of the ridged circular waveguide is investigated using the finite-element technique to show how the introduction of this waveguide can effectively resolve the problem of the $\mathrm{CAD}$ of the tuning screws in dual-mode filters in circular waveguide.
\end{abstract}

\section{INTRODUCTION}

$\mathbf{T}$ HE POSSIBILITY of implementing dual-mode filters in circular waveguides has been known for some time [1]. This very compact implementation of microwave filters is essentially based on the use of degenerate modes in circular waveguide resonators. The key elements of this type of filters are the input/output couplings, the inter resonator irises, and the "tuning screws assembly" (TSA). The use of tuning screws is very common for the implementation of many types of microwave filters. Their function is generally to allow for "tuning" of the electrical characteristics of the hardware that is necessary to compensate for the non ideal machining of the hardware or for the inaccuracy of the electromagnetic modeling available.

For many filter types, the availability of accurate electromagnetic modeling and precision machining can completely eliminate the need for tuning screws. For dual-mode filters, however, this is not the case. In fact, the TSA in a dualmode filter has a fundamental role that is not related to "inaccuracies." In a typical implementation, one physical cavity must support two degenerate electrical resonances that may be loaded electrically in a different way and that may need, in addition, to be coupled to each other. To obtain the same resonance frequency two tuning screws are introduced, at 90 degrees from each other. To introduce the coupling a third screw is introduced at an appropriate locations. The screws are therefore an integral part of the filter and can not be eliminated. On the other hand, their electromagnetic modeling is not a trivial task.

Manuscript received July 24, 1992.

The authors are with the European Space Research and Technology Centre (ESTEC), P.O. Box 299, 2200 AG Noordwijk, The Netherlands.

IEEE Log Number 9204119.

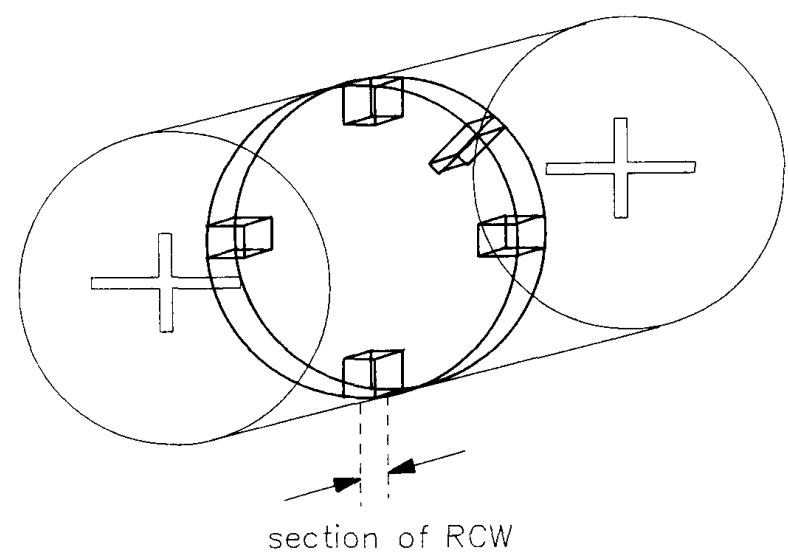

Fig. 1. Dual-mode cavity using a section of ridged circular waveguide.

Recently a dual-mode filter implementation has been proposed in microstrip which is based on the use of ring resonators [2]. The equivalent function of the TSA in the structure proposed in [2] is performed by two pairs of stubs (degenerate resonances tuning) and by an additional radial-stub discontinuity (coupling). In complete analogy with the microstrip implementation, we propose to introduce in the center of a dual-mode resonators in circular waveguide a section of uniform ridged circular waveguide (RCW) with two pairs of ridges for the tuning and a fifth ridge to accomplish the coupling between resonances, as shown in Fig. 1. The full wave modeling of the TSA is then reduced to the much easier problem of the transition between two uniform waveguides.

\section{RidGed Circular Waveguide}

The problem of eliminating the manually adjusted TSA in a dual-mode filter in circular waveguide has been reduced to the study of the step-transition between a uniform circular waveguide and a ridged circular waveguide. This particular decomposition of the problem allows for the development of useful CAD tools based on modal analysis for the simulation of the complete filter structures. The key element now becomes the knowledge of the modal structure of the ridged circular waveguide.

The study of such structure can be conveniently carried out by using the finite-element technique for the solution of the modal equations [3], which can be conveniently rephrased in the form

$$
\int_{\Gamma} w \vec{\nabla}_{t} \phi \cdot \vec{n} d \Gamma-\int_{\Omega} \vec{\nabla}_{t} w \cdot \vec{\nabla}_{t} \phi d \Omega+k_{t}^{2} \int_{\Omega} w \phi d \Omega=0
$$



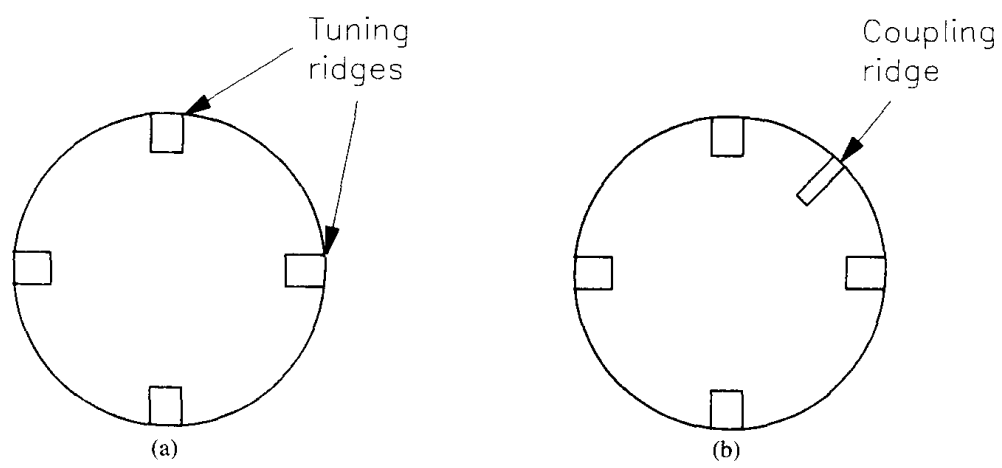

Fig. 2. Cross section of RCW. (a) Without "coupling ridge." (b) With "coupling ridge."

where $\Omega$ is the domain where a solution to the problem is sought (the waveguide cross-section), $\Gamma$ is its boundary and $w$ is a suitable weighting function. This weighted residual statement $[4]$ is then implemented by a finite-element discretization. More details in this context will be published in a forthcoming publication describing the CAD of complete filters.

The following are three essential features that the RCW must exhibit in order to be conveniently used in circular waveguide dual-mode filters.

- It must support the propagation of degenerate modes.

- It must be possible to change independently the wavenumbers of the degenerate modes.

- It must be possible to introduce the proper coupling between the degenerate modes.

The RCW exhibits all of these essential features.

Table I shows the computed transverse eigenvalues for the structure in Fig. 2(a) for the first 16 modes. As we can see from Table I, the structure can support degenerate sets of modes as indicated by solutions with the same eigenvalues. Furthermore, Table II shows the effect of a change in penetration of one pair of "tuning ridges" (Fig. 2(a)) clearly indicating that the individual (degenerate) modes can be "tuned." Additional information is shown in Table III where we present the results of the computations of the transverse eigenvalues of the fundamental TE degenerate modes as a function of the penetration of the fifth ridge for the structure in Fig. 2(b) (the "coupling ridge") while all other "tuning" ridges are kept equal. The separation of the numerical values of the eigenvalues clearly indicates that the two degenerate modes are being coupled by the presence of the fifth ridge.

\section{CONCLUSION}

A solution to the problem of eliminating the manually adjusted "tuning screws assembly" from a dual-mode filter in circular waveguide has been proposed with the introduction of a new ridged circular waveguide ( $\mathrm{RCW}$ ). By following the approach described in this letter, the initial problem has been reduced to the study of the modal structure of the $\mathrm{RCW}$. Initial results concerning the modal spectrum of this waveguide have been presented which are based on the use of the finiteelements techniques and indicate that the proposed method is indeed effective. Further work is currently in progress with the development of a CAD tool for the simulation of complete
TABLE I

Transverse EigenVAlues for the First 8 TE AND TM MODES OF THE RCW IN Fig. 2(a)

\begin{tabular}{ccc}
\hline & TE & TM \\
\hline$n=1$ & 1.8279 & 2.6435 \\
$n=2$ & 1.8279 & 4.1631 \\
$n=3$ & 2.6679 & 4.1631 \\
$n=4$ & 3.3577 & 5.2129 \\
$n=5$ & 3.8720 & 5.8722 \\
$n=6$ & 4.0032 & 5.9444 \\
$n=7$ & 4.0032 & 6.7116 \\
$n=8$ & 4.5129 & 6.7116 \\
\hline
\end{tabular}

Four ridges in Fig. 2(a) are $15^{\circ}$ wide and $0.2 a$ deep ( $a$ is the radius of the RCW).

TABLE II

Percentage Variation of the Transverse Eigenvalues of the LOWEST "DEGENERATE" TE MODES FOR THE RCW IN FIG. 2(a) AS A Function of the Penetration of One Pair of "Tuning Ridges"

\begin{tabular}{cccccc}
\hline & $d / a=-0.1$ & $d / a=-0.05$ & $d / a=0.0$ & $d / a=0.05$ & $d / a=0.1$ \\
$(\%)$ & $(\%)$ & $(\%)$ & $(\%)$ & $(\%)$ \\
\hline$n=1$ & 3.2075 & 1.4701 & 0.0000 & -1.3030 & -2.2720 \\
$n=2$ & -6.1143 & -3.5082 & 0.0000 & 4.5439 & 10.2573 \\
\hline
\end{tabular}

Depth of one pair of ridges is kept the same as in Table I. Depth of the others is varied. $d / a$ indicates the normalized variation.

TABLE III

Percentage Variation of the Transverse Eigenvalues of the LOWEST "DEgenerate” TE MODES For THE RCW IN FIG. 2(b) AS A Function of the "Coupling Ridge" Relative Penetration $c / a$

\begin{tabular}{cccccc}
\hline & $c / a=0.05$ & $c / a=0.10$ & $c / a=0.15$ & $c / a=0.20$ & $c / a=0.25$ \\
& $(\%)$ & $(\%)$ & $(\%)$ & $(\%)$ & $(\%)$ \\
\hline$n=1$ & 0.0167 & 0.2673 & 1.0023 & 1.9044 & 3.1741 \\
$n=2$ & -0.2673 & -0.5346 & -1.1694 & -1.5035 & -1.8376 \\
\hline
\end{tabular}

"Tuning" ridges are kept as in Table I. "Coupling" ridge is $5^{\circ}$ wide.

dual-mode filters based on the approach described.

\section{REFERENCES}

[1] A. E. Atia and A. E. Williams, "New types of waveguide band pass filters for satellite transponders, Comsat Tech. Rev. 1, no. 1, pp. 21-42, 1971

[2] M. Guglielmi and G. Gatti, "Experimental Investigation of Dual-Mode Microstrip Ring Resonators," Proc. 20th European Microwave Conf., Budapest, Hungary, Sept. 1990, pp. 901-906.

[3] N. Marcuvitz, Waveguide Handbook. New York: McGraw-Hill, 1951

[4] P. A. Raviart and J. M. Thomas, Introduction à l'Analyse Numérique des Equations aux Dérivées Partielles. Paris: Masson, 1983. 